\title{
CADERNOS UnIFOA
}

\section{Análise do risco cardiovascular de pacientes atendidos no ambulatório de cardiologia da policlínica do UniFOA}

\section{Cardiovascular risks analysis in patients attended at the cardiology ambulatory in UniFOA polyclinic}

\author{
1 Tassia Silva Leite tassialeite02@gmail.com \\ 1 Rhanna Teixeira de Souza \\ 1 Joselaine dos Santos Cerqueira \\ 2 Leandro de Paula Martins \\ 1 Discente do curso de Medicina - Centro Universitário de Volta Redonda - UniFOA \\ 2 Especialista em Cardiologia UniFOA - Centro Universitário de Volta Redonda
}

\section{Resumo}

As doenças cardiovasculares representam a principal causa de morbimortalidade no Brasil e no mundo. Está bem estabelecida a relação entre o desenvolvimento das doenças cardiovasculares e os fatores de risco, como dislipidemia, tabagismo, sedentarismo e obesidade, principalmente a obesidade central, que são fatores de risco modificáveis. Nas últimas décadas, observam-se diversas transformações no padrão de morbimortalidade, em decorrência dos processos de transição demográfica, epidemiológica e nutricional, bem como em virtude das mudanças no estilo de vida da sociedade moderna. Devido a isso, o objetivo desta pesquisa foi avaliar o risco cardiovascular, com ênfase nas variáveis: diabetes mellitus, dislipidemia, hipertensão arterial e tabagismo de uma população da cidade de Volta Redonda atendida na Policlínica Professor André Bianco, do UniFOA. Foi feita uma análise dos prontuários desses pacientes; posteriormente o risco cardiovascular foi calculado e os dados foram tabulados. A maioria dos pacientes teve risco moderado ou alto e as variáveis mais prevalentes foram dislipidemia e hipertensão arterial. A maior parte dos fatores de risco cardiovascular analisados neste estudo é modificável, por isso, reforça-se a importância de incentivar a adoção de um estilo de vida mais saudável nessa população, além de ser fundamental um elo entre políticas de saúde e a comunidade e a Estratégia de Saúde da Família como estrutura potencial na consolidação da promoção da saúde.

\section{Palavras-chave}

Risco cardiovascular. Doenças cardiovasculares. Fatores de risco. Estilo de vida saudável.

\begin{abstract}
Cardiovascular diseases represent the main cause of morbidity and mortality in Brazil and worldwide. The relationship between the development of cardiovascular diseases and risk factors, such as dyslipidemia, smoking, physical inactivity and obesity, especially central obesity, which are modifiable risk factors, is well established. In recent decades, several changes in the pattern of morbidity and mortality have been observed, due to the demographic, epidemiological and nutritional transition processes, as well as to changes in the lifestyle of modern society. Due to this, the objective of this research was to evaluate cardiovascular risk, with emphasis on the variables: diabetes mellitus, dyslipidemia, hypertension and smoking in Volta Redonda population attended at Policlínica Professor André Bianco, in UniFOA. An analysis of the medical records of these patients was made; cardiovascular risk was subsequently calculated and data were tabulated. Most patients had moderate or high risk and the most prevalent variables were dyslipidemia and hypertension. Most of the cardiovascular risk factors analyzed in this study are modifiable, therefore, the importance of encouraging the adoption of a healthier lifestyle in this population is reinforced, and a link between health policies and the community is fundamental and the Family Health Strategy as a potential structure in the consolidation of health promotion.
\end{abstract}

\section{Keywords}

Cardiovascular risk. Cardiovascular diseases. Risk factors. Healthy lifestyle.

\section{Como você deve citar?}

LEITE, Tassia Silva et al. Análise do risco cardiovascular de pacientes atendidos no ambulatório de cardiologia da policlínica do UniFOA. Cadernos UniFOA, Volta Redonda, n. 44, p. 133-143, dezembro 2020. 


\section{INTRODUÇÃO}

Risco cardiovascular é definido como a probabilidade que uma pessoa tem de adquirir uma doença cardiovascular no futuro. As doenças cardiovasculares representam a principal causa de morbimortalidade no Brasil e no mundo. Esse cenário epidemiológico preocupa por implicar na diminuição da qualidade de vida das populações e em custos elevados e crescentes para governo, sociedade, família e indivíduos (CARVALHO et al., 2015).

O conjunto de doenças crônico-degenerativas é a principal causa de internações hospitalares no setor público pela população adulta, respondendo por $16,22 \%$ do gasto total do Sistema Único de Saúde (SUS) como a maior causa de gastos em assistência médica (OLIVEIRA et al., 2013)

Está bem estabelecida a relação entre o desenvolvimento das doenças cardiovasculares e os fatores de risco, como dislipidemia, tabagismo, sedentarismo e obesidade, principalmente a obesidade central, que são fatores de risco modificáveis. Por muito tempo, esses fatores foram considerados importantes apenas em populações com idade avançada. Entretanto, ultimamente, os estudos têm demonstrado que já são uma realidade entre adultos jovens e até mesmo entre crianças e adolescentes (CARVALHO et al., 2015).

Nas últimas décadas, observam-se diversas transformações no padrão de morbimortalidade, em decorrência dos processos de transição demográfica, epidemiológica e nutricional, bem como em virtude das mudanças no estilo de vida da sociedade moderna (PINHO et al., 2014).

Devido a isso, a exposição a fatores de risco cardiovascular em nosso cotidiano é comum. 0 ritmo de vida da sociedade contemporânea, o consumismo e a mídia convidam para uma omissa e curiosa relação de prazer e riscos entre os jovens e o uso de álcool, a pouca atividade física, os fast foods, dentre outras, tornando corriqueira a vulnerabilidade à doença cardiovascular (GOMES et al., 2012).

Além disso, modificações ocorridas no cenário demográfico brasileiro resultaram em aumento substancial do número de idosos. Dados mostram que o envelhecimento populacional trouxe problemas de saúde que desafiam os sistemas de atendimento e de previdência social. Com isso, é essencial o investimento em programas de prevenção ao longo da vida, visando resolver os desafios atuais que podem refletir no futuro (MIRANDA; MENDES; SILVA, 2016) Essas mudanças acompanham modificações no perfil nutricional da população, com redução na ocorrência da desnutrição, ao mesmo tempo em que se registra o aumento crescente da obesidade. Paralelamente, observam-se alterações no perfil epidemiológico, como a redução das doenças infecciosas e aumento crescente das doenças crônicas não transmissíveis (PINHO et al., 2014).

O número de pessoas que morrerão por doença cardiovascular, principalmente doença cardíaca e acidente vascular encefálico, é estimado em cerca de 23,3 milhões, em 2030, permanecendo como a principal causa de morte no mundo. A Organização Mundial da Saúde (OMS) estima que três quartos da mortalidade cardiovascular possam ser diminuídos com adequadas mudanças no estilo de vida. Esse é o grande desafio das diversas diretrizes existentes em prevenção cardiovascular (JARDIM et al., 2014).

Nessa perspectiva, o uso de aplicativos que calculam o risco cardiovascular se tornou comum nos consultórios de Cardiologia. O ASCVD Risk Estimator Plus é um aplicativo do American College of Cardiology que usa a ciência recente para avaliar pacientes acima de 40 anos. Esse dispositivo é capaz de calcular o risco cardiovascular do paciente em um intervalo de 10 anos. Essa tecnologia ajuda o médico a criar um plano de redução de riscos, por meio de um monitoramento, evitando a evolução para uma doença cardiovascular (AMERICAN COLLEGE OF CARDIOLOGY, 2013). 
O cardiômetro é uma ferramenta criada pela Sociedade Brasileira de Cardiologia (SBC) que realiza continuamente a contagem do número de mortes por doenças cardiovasculares (DCV) no país. Dados obtidos a partir desse recurso revelam que as DCV estão entre as principais causas de morte no Brasil, totalizando 3.493.459 óbitos, de 2004 a 2014, o que representa 29\% do total. Com isso, é possível dizer que ocorre uma morte a cada 40 segundos no Brasil (SOCIEDADE BRASILEIRA DE CARDIOLOGIA, 2018).

Outro dado importante e relevante em relação ao assunto é que as DCV provocam o dobro de mortes que todos os tipos de câncer juntos, 2 a 3 vezes mais que todas as causas externas (acidentes e violência), 3 vezes mais que as doenças respiratórias e 6,5 vezes mais que todas as infecções, abrangendo também a Síndrome da Imunodeficiência Humana Adquirida (SIDA). Por isso, o tratamento e a prevenção devem ser realizados, visando ao enfrentamento dessa situação (SOCIEDADE BRASILEIRA DE CARDIOLOGIA, 2018).

O objetivo desta pesquisa foi avaliar o risco cardiovascular, com ênfase nas variáveis: diabetes mellitus, dislipidemia, hipertensão arterial e tabagismo de uma população da cidade de Volta Redonda atendida na Policlínica Professor André Bianco, do UniFOA, visando: (a) calcular o risco cardiovascular de cada componente da amostra, por meio do ASCVD Risk Estimator Plus e; (b) traçar um perfil epidemiológico dos pacientes.

O tema é relevante porque sabe-se que as DCVs estão entre as principais causas de morte no Brasil e no mundo. A principal maneira de evitar seu desenvolvimento é combater os fatores de risco modificáveis, como tabagismo, sedentarismo e dislipidemia. Soma-se a isso o fato de esses fatores não estarem restritos apenas à população idosa, uma vez que se observam mudanças de hábito de vida, sejam nutricionais ou relacionados com o nível de exercício físico em diversos grupos etários (POZZAN et. al. 2004).

\section{METODOLOGIA}

A pesquisa foi submetida ao Comitê de Ética do UniFOA e aprovada sob o número de CAAE 94246218.2.0000.5237. Trata-se de estudo descritivo exploratório realizado com os pacientes atendidos no ambulatório de Cardiologia da Policlínica do UniFOA Professor André Bianco, no período de fevereiro de 2016 a junho de 2019.

Como critérios de inclusão, foram selecionados prontuários de pacientes entre 40 e 79 anos de idade. Foram excluídos os prontuários de pacientes sem informações essenciais para o cálculo do risco cardiovascular e com letra ilegível.

Para o cálculo do risco cardiovascular foram analisadas as seguintes variáveis: idade, sexo, raça, pressão arterial, colesterol total, LDL, HDL, história de diabetes, tabagismo, hipertensão em tratamento, uso de estatinas e tratamento com aspirina.

Após a coleta dos dados, os riscos foram calculados no aplicativo ASCVD Risk Estimator Plus e classificados em baixo, médio e alto risco. Se o risco for menor do que $5 \%$, é considerado baixo risco, tanto para homens quanto para mulheres; se o risco for entre $5,1 \%$ até $9,9 \%$ para mulheres, é considerado moderado risco e, acima de $10 \%$, é considerado alto risco. No homem, se o risco for entre $5,1 \%$ até $19,9 \%$, é considerado moderado risco e acima de $20 \%$ é considerado alto risco. Esse risco calculado é o risco de o paciente apresentar doenças cardiovasculares, como infarto, AVE (acidente vascular encefálico) e insuficiência cardíaca nos próximos 10 anos. Em seguida, com os riscos cardiovasculares calculados, os resultados foram tabulados e posteriormente gráficos foram feitos no Excel. 


\section{RESULTADOS}

Foram analisados 82 prontuários de pacientes atendidos no ambulatório de Cardiologia da Policlínica do UniFOA Professor André Bianco, no período de fevereiro de 2019 a junho de 2019, referente a consultas realizadas no período de 2016 a 2019. Desses 82 prontuários avaliados de forma aleatória, respeitando-se os critérios de inclusão e exclusão, 23 são de pacientes do sexo masculino e 59 são de pacientes do sexo feminino.

O risco cardiovascular de cada paciente foi calculado, utilizando-se o aplicativo ASCVD Risk Estimator Plus e depois foram classificados em baixo, moderado ou alto risco.

Em relação ao sexo feminino, as pacientes estudadas tinham entre 40 e 79 anos de idade. Quanto ao risco cardiovascular, 17 mulheres apresentaram baixo risco $(28,8 \%), 21$ mulheres apresentaram moderado risco $(35,6 \%)$ e 21 mulheres apresentaram alto risco $(35,6 \%)$, como apresentado no gráfico 1 .

Gráfico 1 - Risco cardiovascular no sexo feminino.

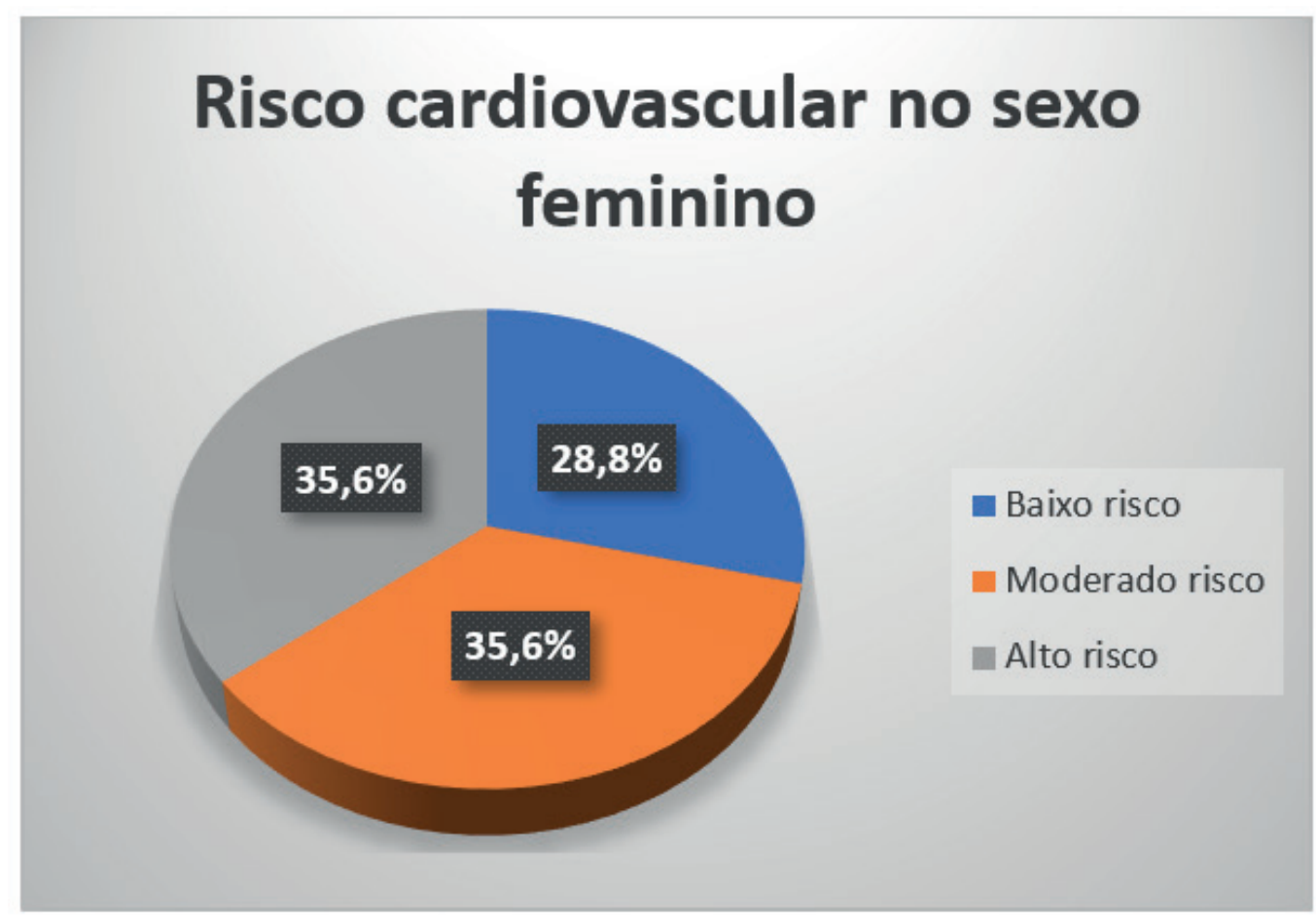

Fonte: dados da pesquisa.

As pacientes que apresentaram baixo risco tinham entre 40 a 65 anos de idade. Dessas, $7(41,1 \%)$ tinham dislipidemia e hipertensão arterial sistêmica (HAS); 6 (35,3\%) tinham apenas HAS; 2 (11,8\%) tinham dislipidemia, HAS e diabetes mellitus; 1 (5,9\%) tinha apenas dislipidemia e 1 (5,9\%) não apresentava nenhuma das variáveis estudadas, como demonstra o gráfico 2. 
Gráfico 2 - Variáveis presentes em mulheres com risco cardiovascular baixo.

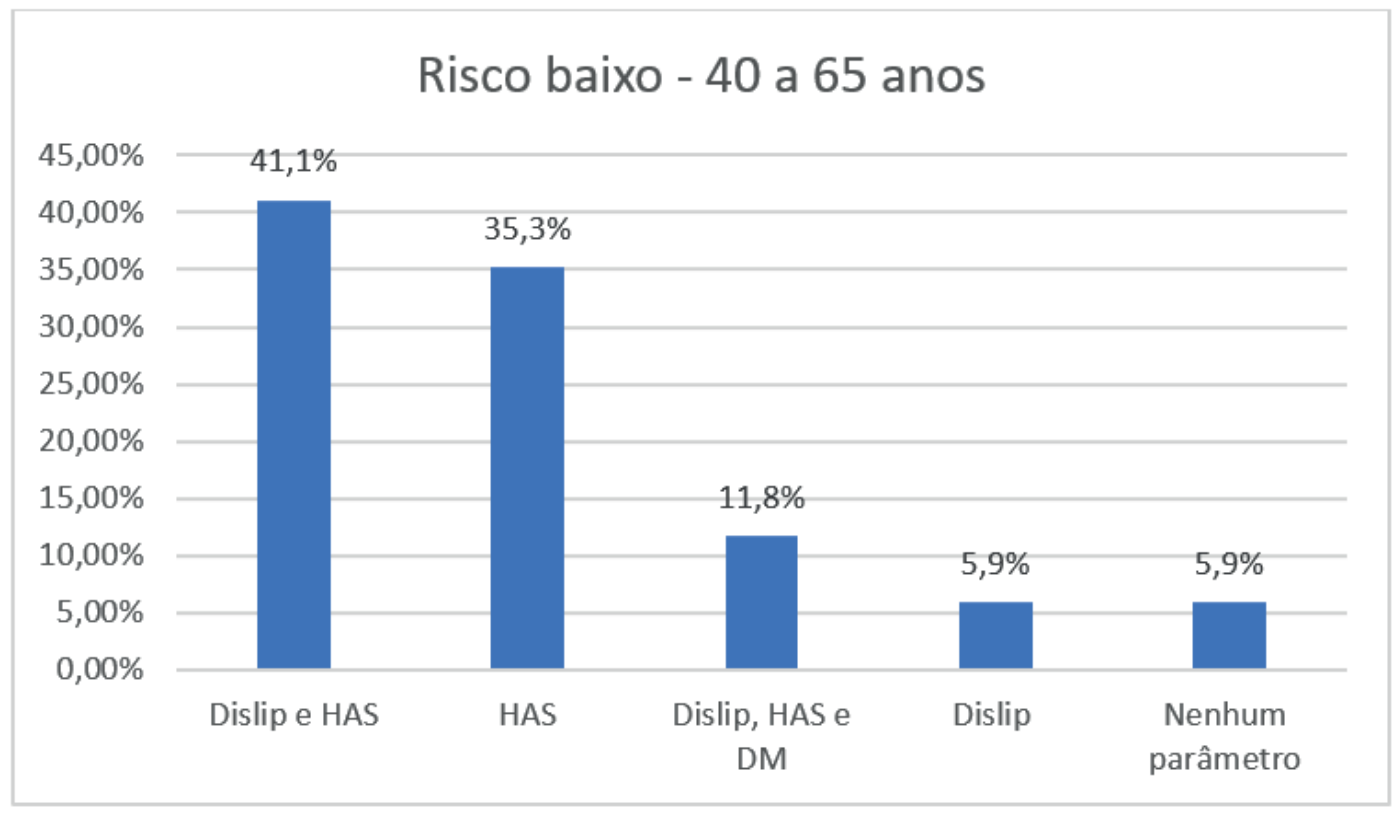

Fonte: dados da pesquisa.

As pacientes que apresentaram moderado risco tinham entre 51 a 70 anos de idade. Dessas, 13 (62\%) tinham dislipidemia e HAS; $3(14,2 \%)$ tinham HAS e diabetes mellitus; $3(14,2 \%)$ tinham dislipidemia, HAS e diabetes mellitus; 1 (4,8\%) tinha dislipidemia, HAS e era tabagista e $1(4,8 \%)$ tinha dislipidemia e diabetes mellitus, como apresentado no gráfico 3.

Gráfico 3 - Variáveis presentes em mulheres com risco cardiovascular moderado.

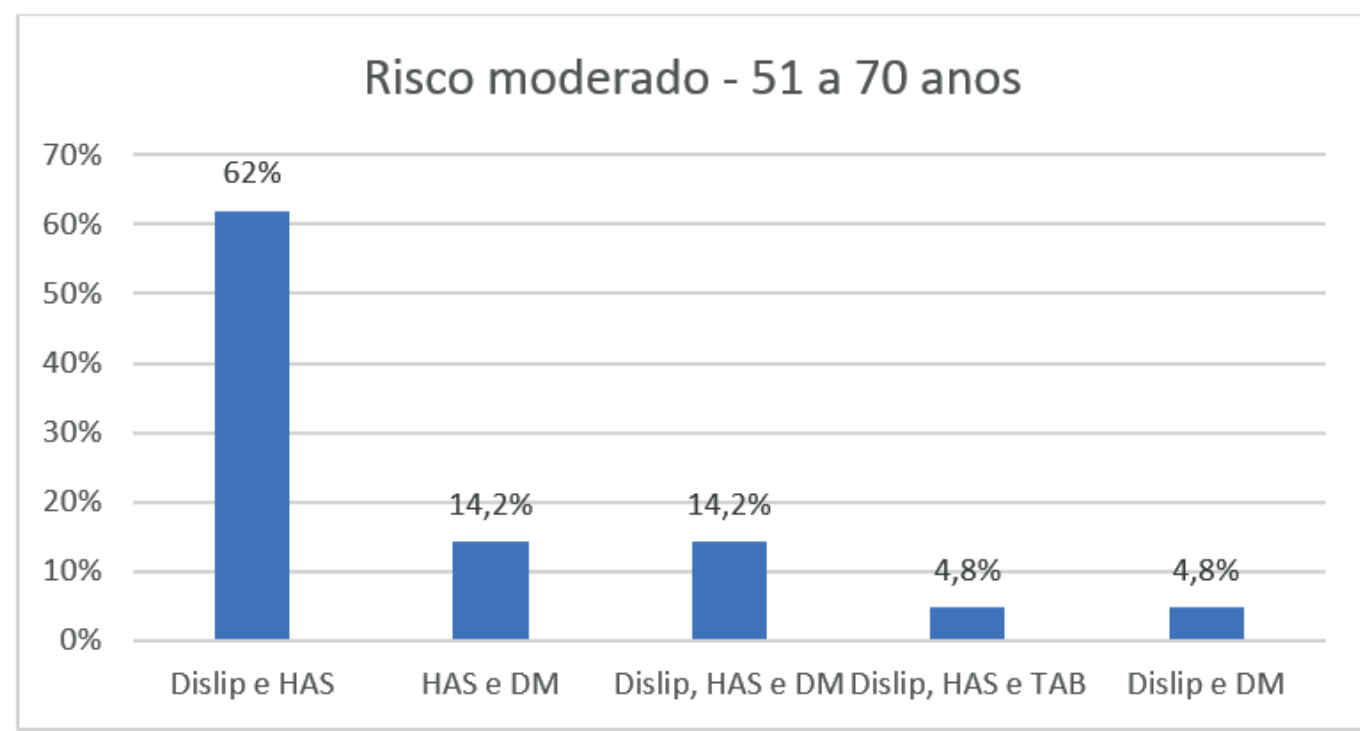

Fonte: dados da pesquisa.

As pacientes que apresentaram alto risco tinham entre 52 a 79 anos. Dessas, $11(52,3 \%)$ tinham dislipidemia e HAS; 7 (33,3\%) tinham dislipidemia, HAS e diabetes mellitus; $1(4,8 \%)$ tinha dislipidemia, 
HAS, diabetes mellitus e era tabagista; 1 (4,8\%) tinha HAS e diabetes mellitus e $1(4,8 \%)$ tinha dislipidemia, HAS e era tabagista, como mostra o gráfico 4.

Gráfico 4 - Variáveis presentes em mulheres com risco cardiovascular alto.

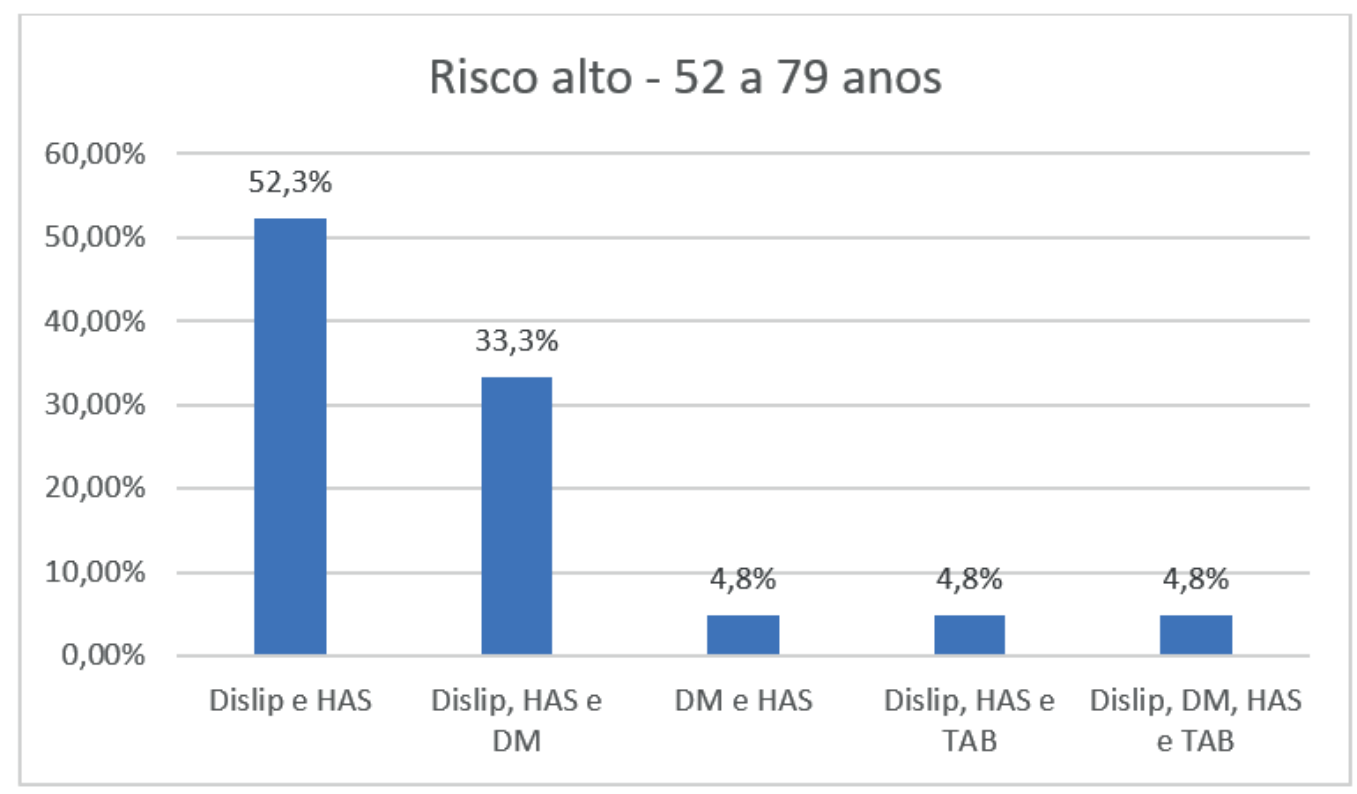

Fonte: dados da pesquisa.

Em relação ao sexo masculino, os pacientes tinham entre 44 e 77 anos de idade. Quanto ao risco cardiovascular, 1 homem apresentou baixo risco (4,4\%), 13 homens apresentaram moderado risco $(56,5 \%)$ e 9 homens apresentaram alto risco (39,1\%), como demonstrado no gráfico 5 .

Gráfico 5 - Risco cardiovascular no sexo masculino.

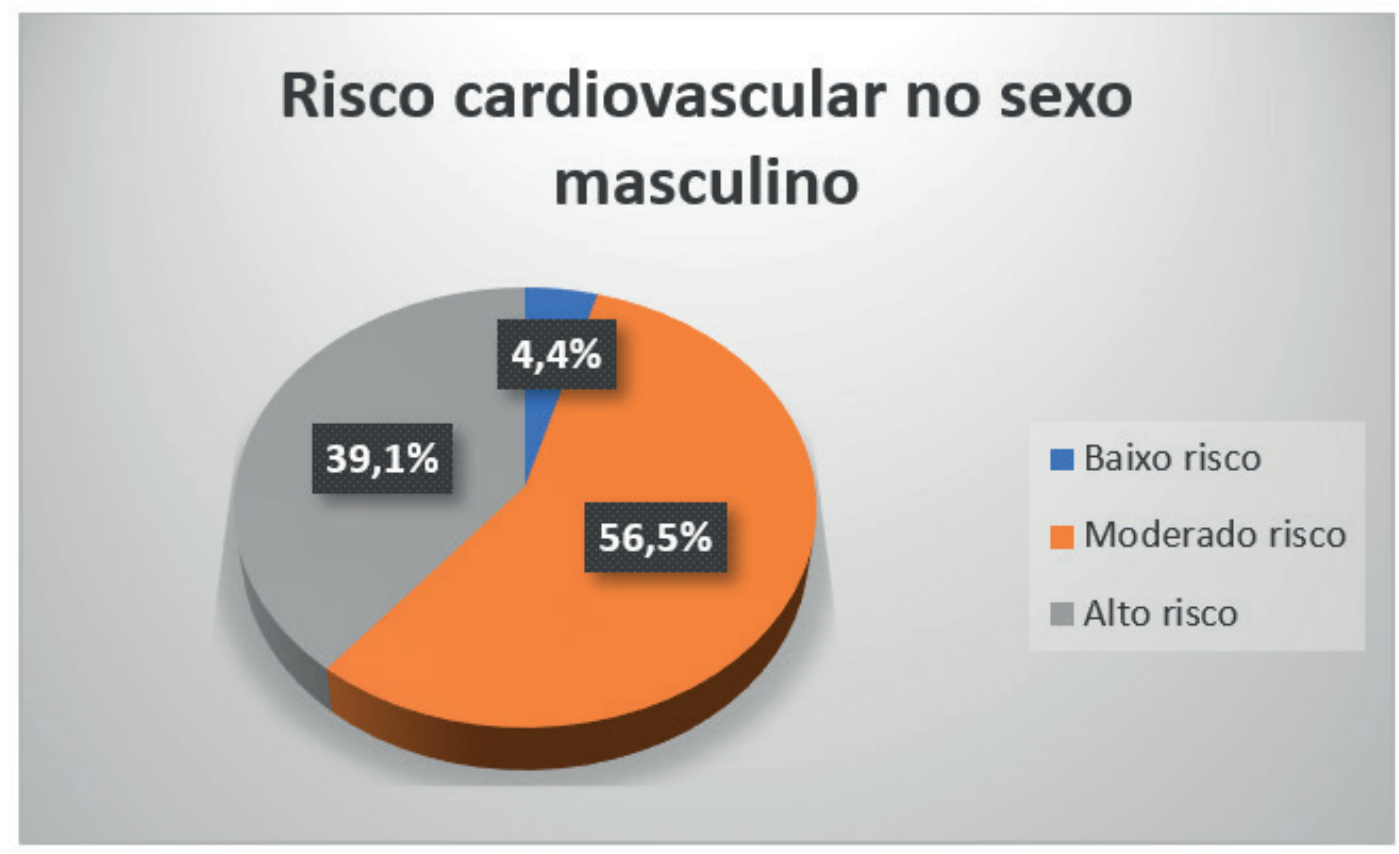

Fonte: dados da pesquisa. 
O único paciente que apresentou baixo risco tinha 44 anos de idade e tinha dislipidemia e HAS.

Os pacientes que apresentaram moderado risco tinham entre 45 a 67 anos de idade. Desses, 11 $(84,6 \%)$ tinham dislipidemia e HAS; $1(7,7 \%)$ tinha dislipidemia, HAS e diabetes mellitus e $1(7,7 \%)$ tinha apenas dislipidemia, como mostra o gráfico 6 .

Gráfico 6 - Variáveis presentes em homens com risco cardiovascular moderado.

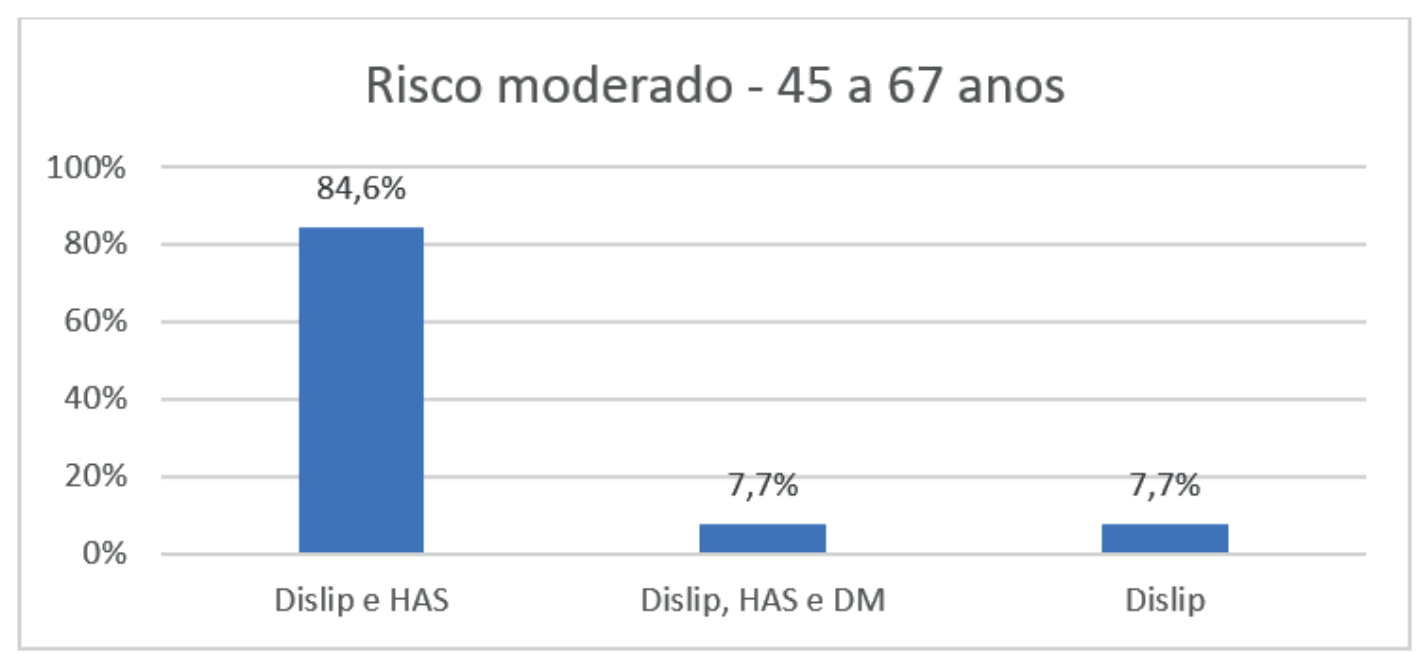

Fonte: dados da pesquisa.

Os pacientes que apresentaram alto risco tinham de 59 a 77 anos de idade. Desses, 6 (66,7\%) tinham dislipidemia, HAS e diabetes mellitus; $1(11,1 \%)$ tinha dislipidemia, HAS e era tabagista; $1(11,1 \%)$ tinha dislipidemia, HAS, diabetes mellitus e era tabagista e $1(11,1 \%)$ tinha dislipidemia e HAS, como mostra o gráfico 7 .

Gráfico 7 - Variáveis presentes em homens com risco cardiovascular alto.

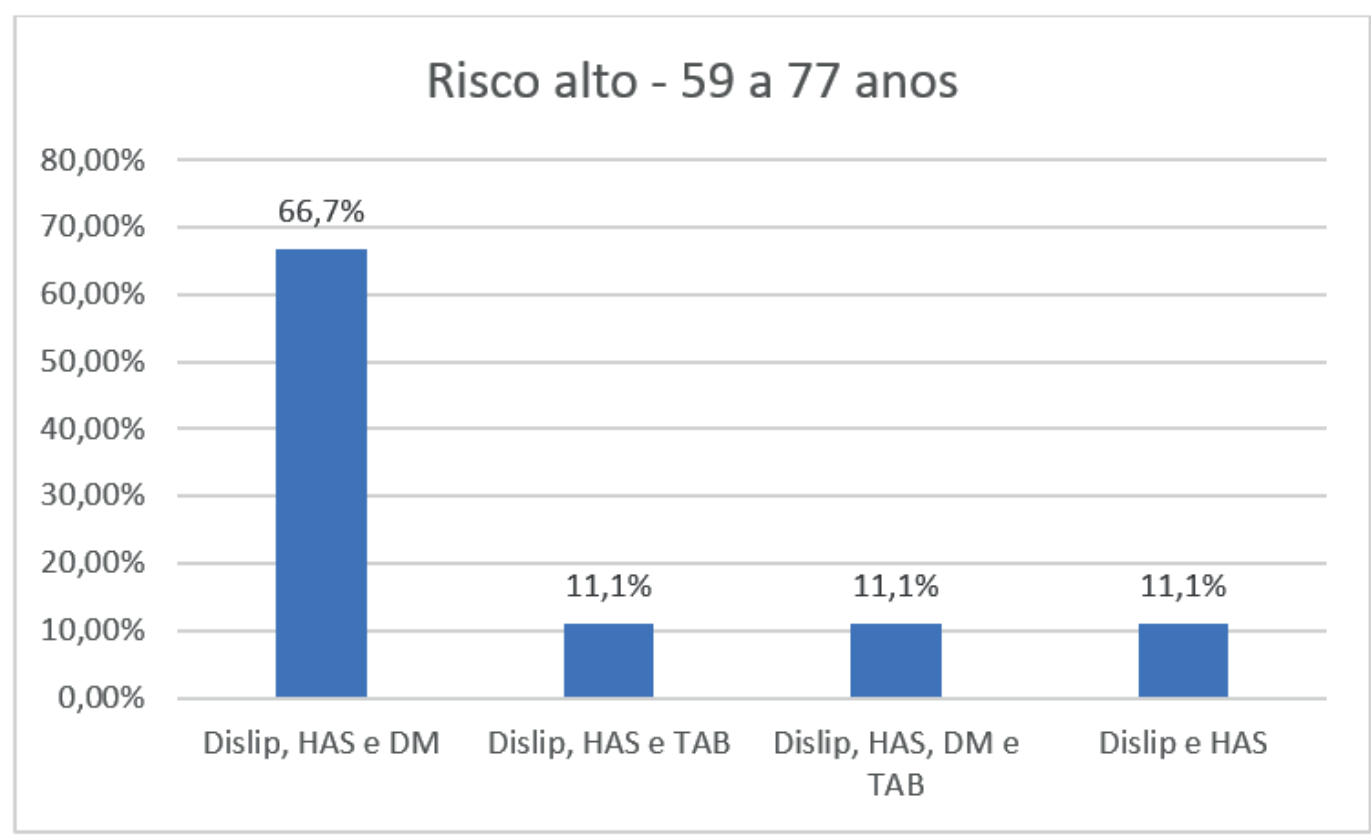

Fonte: dados da pesquisa. 


\section{DISCUSSÃO}

Nesta pesquisa, foi observado que a maioria dos pacientes atendidos no ambulatório de Cardiologia da Policlínica do UniFOA Professor André Bianco, independentemente do sexo, possuía risco cardiovascular moderado ou alto. Isso pode ser explicado pelo fato de a maioria das doenças estar relacionada com a alimentação, atividades diárias e seu ambiente físico e social. Esses dados indicam que provavelmente a população estudada, mesmo com tratamento farmacológico adequado, não está mudando no seu estilo de vida, dificultando a prevenção de possíveis doenças cardiovasculares.

Mesmo a escolha dos prontuários sendo feita de forma aleatória, respeitando os critérios de inclusão e exclusão, houve uma maior prevalência do sexo feminino no estudo. Uma possível explicação é o fato de as mulheres procurarem mais os serviços de saúde, sugerindo uma maior preocupação delas com a saúde ou maior disponibilidade e facilidade de acesso a eles. De acordo com Gomes, Nascimento e Araújo (2007), os homens procuram menos os serviços de saúde, devido a uma cultura que dificulta a adoção de práticas de autocuidado, uma vez que o homem é visto como viril, invulnerável e forte e procurar assistência médica poderia associá-lo à fraqueza, medo e insegurança.

Também foi observado que, quanto maior a idade do paciente, maior o seu risco cardiovascular, pois, como descrito pelos autores Costa, Wagner e Oliveira (2012), o avanço da idade promove mudanças fisiológicas, psicológicas e ambientais que influenciam no estado nutricional e metabólico dos idosos, facilitando o desenvolvimento de doenças cardiovasculares e cerebrovasculares. A idade também representa o fator de risco para doença cardiovascular de maior importância nos idosos, devido às alterações endoteliais que facilitam a ação de outros fatores aterogênicos.

Outro fator observado foi uma maior prevalência de hipertensão arterial sistêmica e dislipidemia na população analisada, sendo esses dois fatores de risco independente do sexo e da estratificação do risco cardiovascular. Segundo Cipullo et. al. (2009), a hipertensão arterial sistêmica é a mais prevalente de todas as doenças cardiovasculares, afetando mais de 36 milhões de brasileiros adultos, sendo o maior fator de risco para doenças cardíacas e cerebrovasculares, e a terceira causa de invalidez. Por isso, o controle rigoroso da hipertensão arterial sistêmica se faz necessário, através do uso de medicamentos e mudanças de estilo de vida, com hábitos alimentares saudáveis e prática de atividade física.

A dislipidemia é um dos distúrbios metabólicos mais comuns na sociedade atual que pode causar a aterosclerose, caracterizando-se como fator de risco para doenças e alterações cardiovasculares (FALUDI et al., 2017). A obtenção do alvo terapêutico de acordo com o risco cardiovascular de cada paciente se faz necessário para evitar o surgimento dessas complicações. Segundo a atualização da Diretriz Brasileira de Dislipidemia e Prevenção da Aterosclerose (2017), pacientes com baixo risco devem ter $L D L<130 \mathrm{mg} / \mathrm{dl}$ e NÃO-HDL $<160 \mathrm{mg} / \mathrm{dl}$; pacientes com moderado risco devem ter $\mathrm{LDL}<100$ $\mathrm{mg} / \mathrm{dl}$ e NÃO-HDL $<130 \mathrm{mg} / \mathrm{dl}$ e pacientes com alto risco devem ter $\mathrm{LDL}<70$ e NÃO-HDL $<100 \mathrm{mg} / \mathrm{dl}$.

O diabetes mellitus também foi visto nessa população, mostrando o quanto a sua relação com o risco cardiovascular é importante. De acordo com Schaan e Portal (2004), além de um maior risco para doença cardiovascular (DCV), indivíduos diabéticos com DCV têm pior prognóstico, apresentando menor sobrevida em curto prazo, maior risco de recorrência da doença e de insuficiência cardíaca congestiva e pior resposta ao tratamento cirúrgico.

A relação do tabagismo com o risco cardiovascular também foi evidenciada nesta pesquisa, uma vez que os pacientes tabagistas foram classificados com moderado ou alto risco cardiovascular. Portanto, tal fato corrobora o tabagismo como um importante fator de risco modificável que deve ser valorizado. 
Um aspecto relevante da investigação foi obter informações sobre a magnitude da ocorrência dos problemas de saúde nessa população. Na perspectiva da vigilância da saúde cardiovascular, os resultados apresentados podem contribuir no monitoramento dos fatores de risco modificáveis e na obtenção de indicadores de cobertura dos serviços, de captação e acompanhamento da população estudada.

\section{CONCLUSÃO}

As doenças cardiovasculares estão entre as principais causas de morte no Brasil e no mundo, sendo responsáveis por altos custos diretos e indiretos, além de impor grandes desafios para o setor de saúde e para as políticas públicas envolvidas no combate aos seus fatores de risco. Os resultados apresentados nesta pesquisa evidenciaram uma prevalência importante de risco cardiovascular na população estudada, principalmente pela presença de HAS, dislipidemia, DM e tabagismo, independentes do sexo. A maioria dos fatores de risco cardiovascular analisados neste estudo é modificável, por isso, reforça-se a importância de incentivar a adoção de um estilo de vida mais saudável na população, principalmente em jovens, uma vez que é, ainda na juventude, que o indivíduo adquire vários hábitos que tendem a permanecer durante toda a vida.

É importante ressaltar que, para uma prevenção adequada da doença cardiovascular, é necessária uma boa estratificação do risco e real controle dos fatores predisponentes. Várias diretrizes foram publicadas na tentativa de se prevenir a doença cardiovascular e devem ser seguidas, no entanto se faz necessário associar ao controle sistemático da pressão arterial e do colesterol, o combate ao tabagismo e a prevenção e o tratamento do excesso de peso, da síndrome metabólica e do DM, por intermédio de alimentação adequada e exercício físico. Dessa forma, a atenção básica se mostra como peça fundamental no elo entre políticas de saúde e a comunidade e a Estratégia de Saúde da Família como estrutura potencial na consolidação da promoção da saúde. 


\section{REFERÊNCIAS}

AMERICAN COLLEGE OF CARDIOLOGY, 2013. Disponível em: http://www.acc.org/tools-and-practicesupport/mobile-resources/features/2013-prevention-guidelines-ascvd-risk-estimator. Acesso em: 30 abr. 2018.

CADERNOS DE ATENÇÃO BÁSICA - HIPERTENSÃO ARTERIAL SISTÊMICA - MINISTÉRIO DA SAÚDE, 2006. Disponível em: http://bvsms.saude.gov.br/bvs/publicacoes/caderno_atencao_basica15. pdf. Acesso em: 11 jun. 2018.

CARVALHO, C. A. et al. Associação entre fatores de risco cardiovascular e indicadores antropométricos de obesidade em universitários de São Luís, Maranhão, Brasil. Ciência \& Saúde coletiva, v. 20, n.2, p. 479-490, 2015.

CAVARARO, R. Tabagismo, 2008. Disponível em: https://biblioteca.ibge.gov.br/visualizacao/livros/ liv42980.pdf. Acesso em: 22 maio 2018.

CHOR, D. Saúde pública e mudanças de comportamento: uma questão contemporânea. Caderno de Saúde Pública, v.15, n.2, p. 423-425, 1999.

CIPULLO, J. P. et. al. Prevalência e fatores de risco para hipertensão em uma população urbana brasileira. Arquivos Brasileiros de Cardiologia, v. 96, n.4, 2009.

COOKE, P.; BITTERMAN, H. Nicotine and angiogenesis: a new paradigm for tobacco-related diseases. Ann. Medicine, v. 36, n. 1, p. 33-40, 2004.

COSTA, J.; WAGNER, R.; OLIVEIRA, L. Avaliação do risco cardiovascular em idosos residentes em asilos da grande Curitiba - PR. Cadernos da escola de saúde, n.8, p. 44-60, 2012.

CRYER, P. E. et al. Norepinephrine and epinephrine release and adrenergic mediation of smoking associated hemodynamic and metabolic events. N Engl J Med, v. 295, p. 573-577, 1976.

EYKEN, E.; MORAES, C. Prevalência de fatores de risco para doenças cardiovasculares entre homens de uma população urbana do Sudeste do Brasil. Cadernos de Saúde Pública, v. 25, p. 111-123, 2009.

FALUDI, A. A. et al. Diretriz brasileira baseada em evidências sobre prevenção de doenças cardiovasculares em pacientes com diabetes: posicionamento da Sociedade Brasileira de Diabetes (SBD), da Sociedade Brasileira de Cardiologia (SBC) e da Sociedade Brasileira de Endocrinologia e Metabologia (SBEM), 2017. Disponível em: http://publicacoes.cardiol.br/2014/diretrizes/2017/04_DIRETRIZ_SBD_SBEM. pdf. Acesso em: 22 maio 2018.

GOMES, E. B. et al. Fatores de risco cardiovascular em adultos jovens de um município do Nordeste Brasileiro. Revista Brasileira de Enfermagem, v. 65, n. 4, p. 594-600, 2012.

GOMES, R.; NASCIMENTO, E.; ARAÚJO, F. Por que os homens buscam menos o serviço de saúde do que as mulheres? As explicações de homens com baixa escolaridade e homens com ensino superior. Cadernos de Saúde Pública, v.23, n.3, p.565-574, 2007.

GRASSI, G. et al. Mechanisms Responsible for Sympathetic Activation by Cigarette Smoking in Humans. Circulation, v. 90, n. 1, 1994. 
JARDIM, T. V. et al. Comparação entre fatores de risco cardiovascular em diferentes

áreas da saúde num intervalo de vinte anos. Arquivos Brasileiros de Cardiologia, arquivo online, 2014.

RIBEIRO, A.; COTTA, R.; RIBEIRO, S. A promoção da saúde e a prevenção integrada dos fatores de risco para doenças cardiovasculares. Ciência \& Saúde Coletiva, v. 17, n. 1, 2012.

RIBEIRO, W. A. et al. Adolescência, tabaco, álcool e drogas: uma revisão no olhar preventivo da educação em saúde na ESF, 2018. Disponível em: http://editora.universidadedevassouras.edu.br/index.php/RPU/ article/view/1115. Acesso em: 11 jun. 2018.

MENDES, M. J. F. L. et al. Associação de fatores de risco para doenças cardiovasculares em adolescentes e seus pais. Rev. bras. saúde matern. infant, p. s49-s54, 2006.

MIRANDA, G.; MENDES, A.; SILVA, A. O envelhecimento populacional brasileiro: desafios e consequências sociais atuais e futuras. Revista Brasileira de Geriatria e Gerontologia, v. 19, n. 3, p. 507-519, 2016.

OLIVEIRA, R. A. R. et al. Variáveis bioquímicas, antropométricas e pressóricas como indicadores de risco cardiovascular em servidores públicos. Fisioterapia em movimento, v. 26, n. 2, p. 369-377, 2013.

PINHO, P. M. et al. Síndrome metabólica e sua relação com escores de risco cardiovascular em adultos com doenças crônicas não transmissíveis. Revista da Sociedade Brasileira de Clínica Médica, v. 12, n. 1, p. 22-30, 2014.

PROTOCOLO CLÍNICO E DIRETRIZES TERAPÊUTICAS. Dislipidemia: Prevenção de Eventos Cardiovasculares e Pancreatite. Portaria SAS/MS n²00 fevereiro de 2013, disponível em: http:// portalarquivos2.saude.gov.br/images/pdf/2014/abril/02/pcdt-dislipidemia-livro-2013.pdf. Acesso em: 22 maio 2018.

RADOVANOVIC, C. A. T. et al. Hipertensão arterial e outros fatores de risco associados às doenças cardiovasculares em adultos. Revista Latino-Americana de Enfermagem, v. 22, n. 4, p. 547-553, 2014.

SCHAAN, B.; HARZHEIM, E.; GUS, I. Perfil de risco cardíaco no diabetes mellitus e na glicemia de jejum alterada. Revista de Saúde Pública, v. 38, p. 529-536, 2004.

SOCIEDADE BRASILEIRA DE CARDIOLOGIA. Disponível em: https://www.cardiol.br/. Acesso em: 22 maio 2018.

SOCIEDADE BRASILEIRA DE DIABETES. Conduta Terapêutica no Diabetes Tipo 2: Algoritmo SBD 2017. São Paulo: 2017. Disponível em: http://www.diabetes.org.br/profissionais/images/2017/ POSICIONAMENTO-OFICIAL-SBD-02-2017-ALGORITMO-SBD-2017.pdf. Acesso em: 22 maio 2018. 